\title{
Universality of global dynamics for the cubic wave equation
}

\author{
Piotr Bizoń ${ }^{1}$ and Anıl Zenginoğllu ${ }^{2,3}$ \\ ${ }^{1}$ M. Smoluchowski Institute of Physics, Jagiellonian University, Kraków, Poland \\ 2 Max-Planck-Institut für Gravitationsphysik (Albert-Einstein-Institut), Golm, Germany \\ ${ }^{3}$ Department of Physics, Center for Scientific Computation and Mathematical Modeling, \\ University of Maryland, College Park, MD 20742, USA
}

Received 26 November 2008, in final form 23 July 2009

Published 10 September 2009

Online at stacks.iop.org/Non/22/2473

Recommended by F Merle

\begin{abstract}
We consider the initial value problem for the spherically symmetric, focusing cubic wave equation in three spatial dimensions. We give numerical and analytical evidence for the existence of a universal attractor which encompasses both global and blowup solutions. As a byproduct we get an explicit description of the critical behaviour at the threshold of blowup.
\end{abstract}

Mathematics Subject Classification: 35L67, 35L70, 35Q75

\section{Introduction}

We consider a semilinear wave equation in three spatial dimensions with the focusing cubic nonlinearity

$$
\partial_{t t} \phi-\Delta \phi-\phi^{3}=0 .
$$

Heuristically, the dynamics of solutions of this equation can be viewed as a competition between the Laplacian which tends to disperse the waves and the nonlinearity which tends to concentrate the waves. For small initial data the dispersion 'wins' leading to global solutions which decay to zero as $t \rightarrow \infty$. For large initial data the dispersive spreading is too weak to counterbalance the focusing nonlinearity and solutions blow up in finite time. For each of these generic evolutions the leading asymptotic behaviour is known: small solutions decay as $1 / t^{2}$ at timelike infinity [1,2], while large solutions diverge as $\sqrt{2} /(T-t)$ for $t$ approaching a blowup time $T[3,4]$. The dichotomy of dispersion and blowup brings up the question of what determines the boundary between these two behaviours and, in particular, what is the evolution of critical initial data which lie on the boundary. During numerical investigations of this question we observed that for a large set of initial data the solutions rapidly converge to a universal attractor which is given by a two-parameter family of explicit solutions of equation (1). The aim of this 
paper is to give analytic and numerical evidence for this behaviour (which is rather surprising for a conservative wave equation). The description of the critical dynamics, which motivated our investigations, emerges as a special case.

The paper is organized as follows. In section 2 we recall some basic properties of solutions of equation (1) and we formulate three conjectures about the existence of the attractor. Analytic evidence for these conjectures is given in section 3. After a short description of our numerical methods and tests in section 4 , we present numerical evidence for the conjectures in section 5 . Finally, in section 6 we make some general remarks.

\section{Preliminaries and conjectures}

In this paper we restrict our attention to spherically symmetric solutions $\phi=\phi(t, r)$, so equation (1) reduces to

$$
\partial_{t t} \phi-\partial_{r r} \phi-\frac{2}{r} \partial_{r} \phi-\phi^{3}=0 .
$$

This equation is invariant under the following transformations:

- translation in time $T_{a}$ by a constant $a$

$$
T_{a}: \phi(t, r) \rightarrow \phi(t+a, r)
$$

- scaling $S_{\lambda}$ by a positive constant $\lambda$

$$
S_{\lambda}: \phi(t, r) \rightarrow \frac{1}{\lambda} \phi\left(\frac{t}{\lambda}, \frac{r}{\lambda}\right),
$$

- conformal inversion $I$ (which is an involution)

$$
I: \phi(t, r) \rightarrow \frac{1}{t^{2}-r^{2}} \phi\left(\frac{t}{r^{2}-t^{2}}, \frac{r}{t^{2}-r^{2}}\right),
$$

- reflection $\phi \rightarrow-\phi$.

Neglecting the Laplacian in (1) and solving the ordinary differential equation $\partial_{t t} \phi-\phi^{3}=0$, one gets the one-parameter family of spatially homogeneous solutions

$$
\phi_{b}=\frac{\sqrt{2}}{b-t} .
$$

This family is a special case of the two-parameter family of solutions [5]

$$
\phi_{(a, b)}(t, r)=\frac{\sqrt{2}}{t+a+b\left((t+a)^{2}-r^{2}\right)},
$$

which can be obtained from (6) by the action of conformal inversion $I$ followed by the time translation $T_{a}$. The scaling transformation $S_{\lambda}$ acting on $\phi_{(a, b)}$ only rescales the parameters of the solution without changing its form. Note that the solution $\phi_{(a, b)}(t, r)$ is singular on the two-sheeted hyperboloid $t=-a-1 /(2 b) \pm \sqrt{1 /\left(4 b^{2}\right)+r^{2}}$.

The main result of this paper is the observation that the family (7) is an attractor for a large set of initial data. More precisely, we have the following conjectures about the forward-in-time behaviour of solutions of equation (2) starting from smooth, compactly supported (or suitably localized) initial data (by time reflection symmetry analogous conjectures can be formulated for the backward-in-time behaviour):

Conjecture 1. For any generic globally regular solution $\phi(t, r)$ there exist parameters $(a, b) \in$ $\mathbb{R} \times \mathbb{R}^{+}$and $\kappa= \pm 1$ such that $t^{4}\left(\phi(t, r)-\kappa \phi_{(a, b)}(t, r)\right)$ is bounded for all finite $r$ and $t \rightarrow \infty$. 
Conjecture 2. For any solution $\phi(t, r)$ which blows up at the origin in finite time $T$, there exist parameters $(a, b) \in \mathbb{R} \times \mathbb{R}^{-}$and $\kappa= \pm 1$ such that $(T-t)^{-2}\left(\phi(t, r)-\kappa \phi_{(a, b)}(t, r)\right)$ is bounded for $t \rightarrow T^{-}$inside the past light cone of the blowup point.

Conjecture 3. The borderline between dispersive and blowup solutions, described, respectively, in conjectures 1 and 2 , consists of codimension-one globally regular solutions $\phi(t, r)$ for which there exist a parameter $a \in \mathbb{R}$ and $\kappa= \pm 1$ such that $t^{4}\left(\phi(t, r)-\kappa \phi_{(a, 0)}(t, r)\right)$ is bounded for all finite $r$ and $t \rightarrow \infty$.

The parameters $(a, b)$ for which the above assertions hold will be referred to as optimal.

Remark 1. Conjectures 1 and 2 are refinements of the well-known asymptotic behaviour of solutions of equation (2): $t^{-2}$ decay near timelike infinity in the case of global regularity (for small initial data) and $\sqrt{2} /(T-t)$ growth in the case of blowup (for large initial data), respectively.

Remark 2. Although conjecture 3 is a special limiting case of conjecture 1 , we state it as a separate conjecture to emphasize the critical character of the attractor solution $\phi_{(a, 0)}$. Note that the slowly decaying global solutions described in conjecture 3 are not asymptotically free, i.e. they do not scatter.

Remark 3. The genericity condition in conjecture 1 is essential because, as we shall see below, there do exist non-generic very rapidly decaying globally regular solutions which do not converge to the attractor $\phi_{(a, b)}$.

Remark 4. For very large amplitudes the blowup first occurs on a sphere [4] and then conjecture 2 does not hold.

In the rest of the paper we give evidence for the above conjectures. The evidence is based mainly on numerical simulations; however, before presenting numerics, we will give two analytic arguments: one based on linearized stability analysis and one based on an explicit solution.

\section{Analytic evidence}

\subsection{Linearized stability}

In this section we discuss the linearized stability of the attractor solution $\phi_{(a, b)}(t, r)$. Since linearization commutes with symmetries, we may set $a=b=0$ without loss of generality. We denote the resulting solution by $\phi_{0}$, thus $\phi_{0}=\sqrt{2} / t$. In order to determine the spectrum of small perturbations around this forward self-similar solution we will use the symmetry under conformal inversions. To this end, consider the region $I^{+}(O)$, the interior of the future light cone of the origin $(0,0)$ of the Minkowski spacetime, and its foliation by spacelike hyperboloids (in this paragraph we follow Christodoulou [6])

$$
t=c+\sqrt{c^{2}+r^{2}}
$$

where $c$ is a positive constant. The hyperboloid (8) is asymptotic to $\partial I^{+}(c)$, the future light cone of the point $(c, 0)$. Let us make the conformal inversion

$$
I:(t, r) \mapsto(\bar{t}, \bar{r})=\left(\frac{t}{r^{2}-t^{2}}, \frac{r}{t^{2}-r^{2}}\right)
$$


This transformation maps $I^{+}(O)$ (in the original coordinate system) to $I^{-}(O)$ (the interior of the past light cone of the origin in the barred coordinate system). In particular, the hyperboloids (8) are mapped to spacelike hyperplanes

$$
\bar{t}=-\bar{c}, \quad \text { where } \quad \bar{c}=\frac{1}{2 c} .
$$

Now, consider a global-in-time solution $\phi(t, r)$ inside $I^{+}(O)$. In the barred coordinates we have from (5)

$$
\bar{\phi}(\bar{t}, \bar{r})=\left(t^{2}-r^{2}\right) \phi(t, r) .
$$

It follows from the above paragraph that the study of the asymptotics of $\phi(t, r)$ for $t \rightarrow \infty$ is equivalent to the study of the asymptotics of $\bar{\phi}(\bar{t}, \bar{r})$ for $\bar{t} \rightarrow 0^{-}$. In particular, there is equivalence between linearized perturbations of $\phi_{0}=\sqrt{2} / t$ for $t \rightarrow \infty$ and linearized perturbations of $\bar{\phi}_{0}=\sqrt{2} /(-\bar{t})$ for $\bar{t} \rightarrow 0^{-}$. But the latter have already been determined in $[4,7]$. Namely, it has been shown there that the spectrum of smooth linearized perturbations about the backward self-similar solution $\sqrt{2} /(-\bar{t})$ consists of a discrete set of eigenmodes of the form $(-\bar{t})^{\lambda_{n}} \xi_{n}(y)$, where $y=\bar{r} /(-\bar{t})$ and $\lambda_{0}=-2, \lambda_{1}=0, \lambda_{n}=n(n \geqslant 2)$. More precisely, we have the following eigenmode expansion around $\bar{\phi}_{0}$ :

$\delta \bar{\phi}(\bar{t}, \bar{r})=c_{0}(-\bar{t})^{-2}+c_{1}\left(1-y^{2}\right)+c_{2}(-\bar{t})^{2}\left(1-\frac{2}{3} y^{2}+\frac{1}{5} y^{4}\right)+\mathcal{O}\left((-\bar{t})^{3}\right)$.

By (11), this translates into the following eigenmode expansion around $\phi_{0}$ (where now $y=r / t)$ :

$$
\delta \phi(t, r)=c_{0}\left(1-y^{2}\right)+c_{1} \frac{1}{t^{2}}+c_{2} \frac{1}{t^{4}} \frac{1-2 y^{2} / 3+y^{4} / 5}{\left(1-y^{2}\right)^{3}}+\mathcal{O}\left(1 / t^{5}\right) .
$$

The first two eigenmodes in the expansions (12) and (13) correspond to the perturbations along the symmetry orbits $\bar{\phi}_{(a, b)}(\bar{t}, \bar{r})$ and $\phi_{(a, b)}(t, r)$, respectively. For example, we have

$$
\left.\frac{\partial}{\partial b} \phi_{(a, b)}(t, r)\right|_{(a=0, b=0)} \sim 1-y^{2},\left.\quad \frac{\partial}{\partial a} \phi_{(a, b)}(t, r)\right|_{(a=0, b=0)} \sim \frac{1}{t^{2}} .
$$

The choice of the optimal parameters $(a, b)$ for the attractor amounts to tuning away the coefficients of the symmetry modes $c_{0}$ and $c_{1}$, hence the rate of convergence to the attractor is expected to be governed by the third eigenmode in the expansions (12) and (13):

$$
\bar{\phi}(\bar{t}, \bar{r})-\bar{\phi}_{(a, b)}(\bar{t}, \bar{r}) \sim(-\bar{t})^{2} \quad \text { for } \bar{t} \rightarrow 0^{-}
$$

and

$$
\phi(t, r)-\phi_{(a, b)}(t, r) \sim 1 / t^{4} \quad \text { for } t \rightarrow \infty,
$$

which is consistent with our conjectures. Below we will verify this expectation numerically, but first we want to give a simple example which corroborates (16).

\subsection{The conformal solution}

Equation (2) has the following globally regular explicit solution:

$$
\phi_{\text {conf }}(t, r)=\frac{2}{\sqrt{\left(1+(t-r)^{2}\right)\left(1+(t+r)^{2}\right)}},
$$

which we will refer to as the conformal solution. Note that this solution corresponds to time symmetric initial data

$$
\phi(0, r)=\frac{2}{1+r^{2}}, \quad \partial_{t} \phi(0, r)=0 .
$$



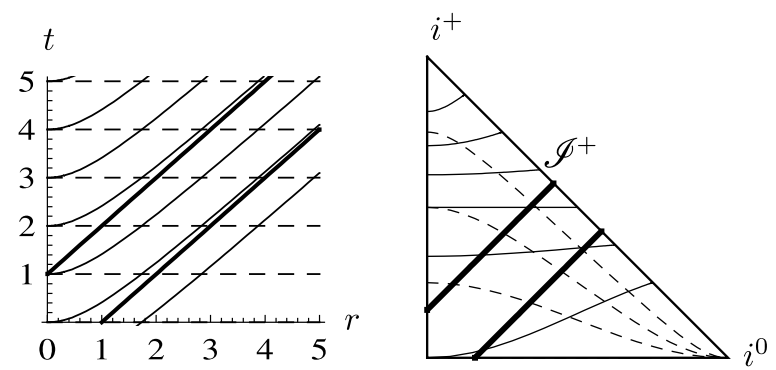

Figure 1. The future domain of a $t=0$ surface is partially depicted on the left panel in standard coordinates and entirely represented on the right panel in a Penrose diagram [8-10]. Dashed lines are level sets of $t$, solid lines are hyperboloids shifted in time as given by (20) and the thick straight lines depict outgoing characteristics indicating the location of the approximate wavefront.

The conformal solution can be easily found in the framework of conformal compactification which maps Minkowski space with the flat metric $\eta$ into the Einstein universe with the conformal metric $g=\Omega^{2} \eta$, where the conformal factor $\Omega=2\left[\left(1+(t-r)^{2}\right)\left(1+(t+r)^{2}\right)\right]^{-1 / 2}[8]$. Under this mapping the cubic wave equation $\square \phi+\phi^{3}=0$ transforms to $\square_{g} \Phi-\Phi+\Phi^{3}=0$, where $\Phi=\Omega^{-1} \phi$. The trivial constant solution $\Phi=1$ gives $\phi_{\text {conf }}=\Omega$.

By elementary calculation we find that for the conformal solution the optimal parameters of the attractor $\phi_{(a, b)}$ are $(a, b)=(-1 / \sqrt{2}, 1 / \sqrt{2})$. More precisely, we have for $t \rightarrow \infty$

$$
\phi_{\text {conf }}(t, r)-\phi_{\left(-\frac{1}{\sqrt{2}}, \frac{1}{\sqrt{2}}\right)}(t, r)=-\frac{1}{t^{4}} \frac{3+y^{2}}{\left(1-y^{2}\right)^{3}}+\mathcal{O}\left(t^{-6}\right),
$$

in agreement with conjecture 1 .

\section{The numerical method and tests}

\subsection{The hyperboloidal initial value problem for the cubic wave equation}

In order to study the asymptotic behaviour of solutions and verify the conjectured convergence to the attractor, it is convenient to foliate Minkowski spacetime by hyperboloids. A time coordinate $\tau$ adapted to such a foliation can be written as

$$
\tau=t-\sqrt{\frac{9}{K^{2}}+r^{2}} .
$$

The level surfaces of $\tau$ are standard hyperboloids shifted in the time direction. They have constant mean curvature $K$ which is a free parameter. In the following we set $K=3$ for simplicity. A foliation of Minkowski spacetime by level sets of $\tau$ is depicted in figure 1 .

In order to be able to analyse the propagation of the outgoing waves to infinity we introduce a compactifying radial coordinate $\rho$ along the surfaces of our foliation. For the regularity of our equations in this compactified setting, we perform a suitable conformal rescaling of the metric. The rescaling factor denoted by $\Omega$ must be a function of $\rho^{2}$ to ensure regularity at the origin in the conformal manifold. We choose $\Omega=\left(1-\rho^{2}\right) / 2$ following [11-13]. The compactifying coordinate is then chosen according to $r=\rho / \Omega$.

We express the standard Minkowski metric $\eta$ in the new coordinates $(\tau, \rho)$ and rescale it with the conformal factor $\Omega^{2}$ to obtain

$$
g=\Omega^{2} \eta=-\Omega^{2} \mathrm{~d} \tau^{2}-2 \rho \mathrm{d} \tau \mathrm{d} \rho+\mathrm{d} \rho^{2}+\rho^{2} \mathrm{~d} \sigma^{2},
$$


where $\mathrm{d} \sigma^{2}$ is the standard metric on the unit two sphere. In these coordinates the zero set of the conformal factor corresponds to future null infinity denoted by $\mathscr{I}^{+}$. We rewrite the cubic wave equation on Minkowski spacetime in conformally covariant form

$$
\square_{g} \Phi-\frac{1}{6} R[g] \Phi+\Phi^{3}=0, \quad \text { where } \quad \Phi=\frac{\phi}{\Omega}, \quad g=\Omega^{2} \eta .
$$

The Ricci scalar of the metric $g$ from (21) appearing in the above equation is given by

$$
R[g]=\frac{12\left(1-\rho^{2}\right)\left(3+\rho^{2}\right)}{\left(1+\rho^{2}\right)^{3}} .
$$

With the auxiliary variables,

$$
\psi:=\partial_{\rho} \Phi \quad \text { and } \quad \pi:=\frac{2}{1+\rho^{2}}\left(\partial_{\tau} \Phi+\rho \partial_{\rho} \Phi\right),
$$

we can rewrite system (22) in first order symmetric hyperbolic form as

$\partial_{\tau} \Phi=\frac{1+\rho^{2}}{2} \pi-\rho \psi$

$\partial_{\tau} \psi=\partial_{\rho}\left(\frac{1+\rho^{2}}{2} \pi-\rho \psi\right)$,

$\partial_{\tau} \pi=\frac{1}{\rho^{2}} \partial_{\rho}\left(\rho^{2}\left(\frac{1+\rho^{2}}{2} \psi-\rho \pi\right)\right)+\frac{1+\rho^{2}}{2}\left(\Phi^{3}-\frac{1}{6} R[g] \Phi\right)$.

The initial data are arbitrary in our conjectures. In the numerical studies presented below we choose a Gaussian pulse

$$
\Phi(0, \rho)=A \mathrm{e}^{-\left(\rho-\rho_{\mathrm{c}}\right)^{2} / \sigma^{2}}, \quad \partial_{\tau} \Phi(0, \rho)=0,
$$

with fixed parameters $\rho_{\mathrm{c}}=0.3, \sigma=0.07$, and varying amplitude $A$. Tests for different initial data give the same qualitative results which makes us feel confident that the phenomena described below are universal.

\subsection{The code}

We solve the hyperboloidal initial value problem (23) and (24) numerically using 4th order Runge-Kutta integration in time and 6th order finite differencing in space. At the origin we apply the regularity condition $\psi(\tau, 0)=0$. No boundary conditions are needed at the outer boundary because there are no incoming characteristics due to the compactification. One-sided finite differencing is applied on the numerical boundaries.

To test the code we performed a three level convergence study with 200, 400 and 800 grid cells on the coordinate domain $\rho \in[0,1]$ that has infinite physical extent. For this study, we used an amplitude of $A=2$ which is below the critical amplitude, and a Courant factor of $\Delta \tau / \Delta \rho=0.8$. The convergence factors shown in figure 2 indicate that, as expected, our code is 6 th order convergent after a short transient phase. The dashed curve has been calculated using a much smaller Courant factor of 0.08 to show that the initial transient phase is due to numerical errors in the time integration that converge with 4 th order. The long time convergence plot on the right panel in figure 2 shows that convergence is lost at about $\tau=1000$ for the number of cells used in this study. This is due to accumulation of numerical errors and occurs at later times in tests with higher resolution. We give numerical evidence for universal dynamics only in the convergent regime. Two-level convergence tests using the explicit conformal solution (17) give the same qualitative results.

Further tests can be performed using known properties of solutions to the cubic wave equation as studied in [4]. First, it is known that generic small solutions decay as $t^{-2}$ near 

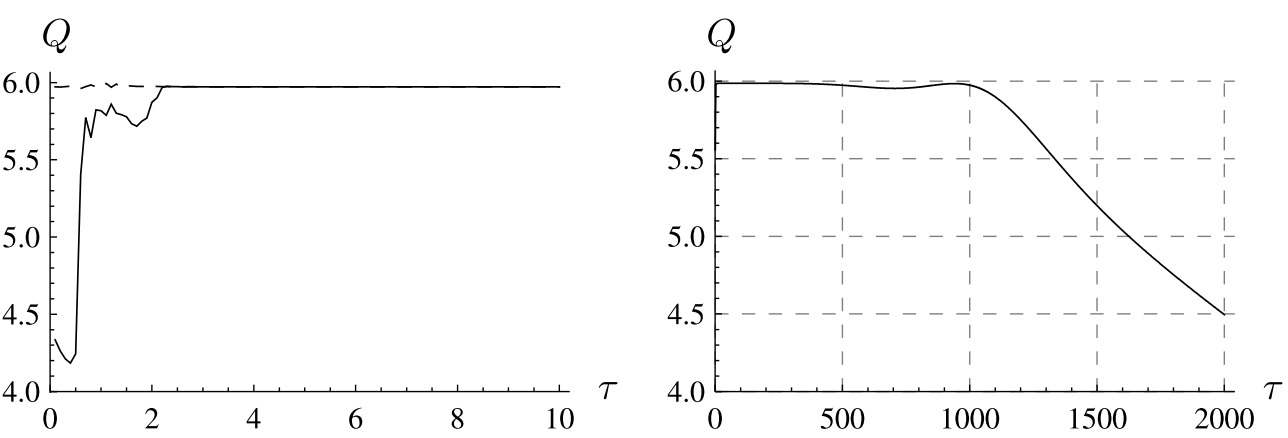

Figure 2. Convergence factors in time measured in the $L_{2}$-norm. The convergence factor $Q$ is defined by $Q=\log _{2} \frac{\left\|\Phi^{\text {low }}-\Phi^{\text {med }}\right\|}{\left\|\Phi^{\text {med }}-\Phi^{\text {high }}\right\|}$. On the left panel we see that after a short transient phase the code converges with 6 th order. The dashed curve corresponds to a simulation with a 10 times smaller Courant factor and shows 6 th order convergence from the beginning. The right panel shows results of a long time convergence test and indicates loss of convergence after about $\tau=1000$.
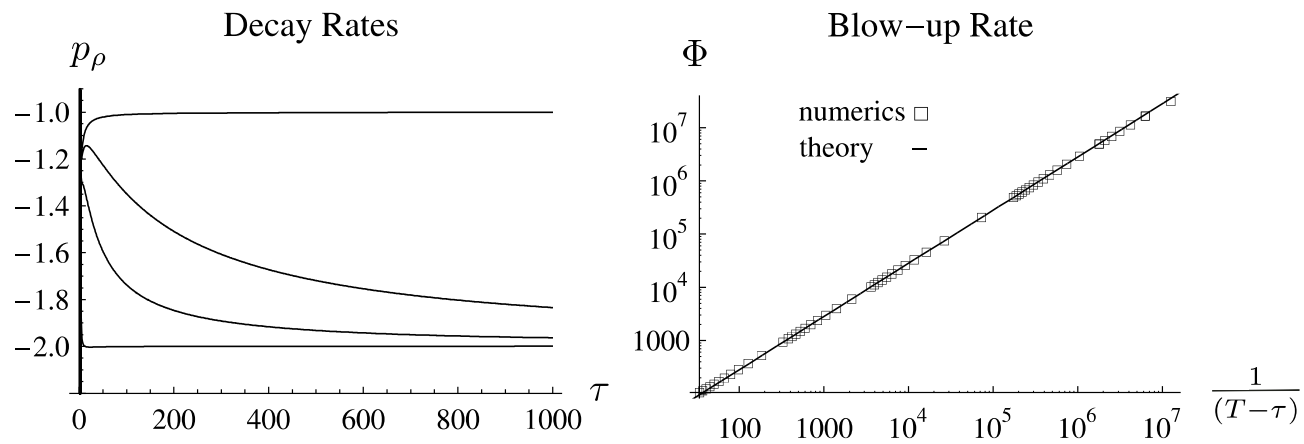

Figure 3. Left panel: the local power index calculated along $\mathscr{I}^{+}, r=\{100,20\}$ and at the origin from top to bottom. Right panel: a numerical solution that blows up at the origin is depicted by small squares and the theoretical prediction of the blowup is depicted by the solid line on a log-log scale. The small squares are not distributed uniformly in time because we reduce our time steps while the solution grows. The figures indicate that the code reproduces the known decay rates for small solutions and the blowup rate for large solutions very accurately.

timelike infinity and $t^{-1}$ along null infinity [2]. It is clear from (7) that the attractor solution has this behaviour for $b>0$. In order to accurately measure the decay rate of the solution along null infinity, near timelike infinity and in the transition domain between these two asymptotic regimes, we calculate the local power index, $p_{\rho}(\tau)$, defined by

$$
p_{\rho}(\tau)=\frac{\mathrm{d} \ln |\Phi(\tau, \rho)|}{\mathrm{d} \ln \tau}
$$

Figure 3 shows that our code reproduces the predicted decay rates very accurately.

Second, it is known that large initial data lead to blowup in finite time with the blowup rate at the origin $\sqrt{2} /(T-t)[3,4]$. This is in accordance with the attractor solution (7) for $b<0$. The right panel in figure 3 shows the numerical solution at the origin for large data on a log-log scale against the theoretical prediction of the blowup rate. The two curves match over many orders of magnitude indicating that our code can reliably handle the blowup. 

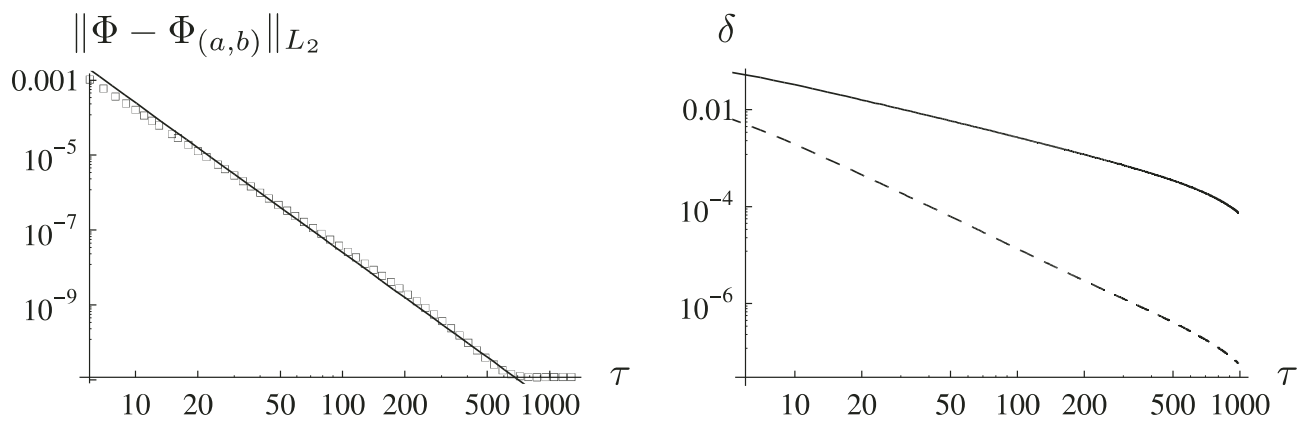

Figure 4. Left panel: the $L_{2}$-norm of the difference between a generic decaying numerical solution and the fitted attractor solution is depicted by the small squares on a log-log scale. The solid line, shown for comparison, has the slope -4 , in accordance with conjecture 1 . Right panel: modulation in the relative errors $\delta$ of the parameters $a$ (solid line) and $b$ (dashed line) on a log-log scale. The modulation for $a$ seems to decay as $t^{-1}$ and for $b$ as $t^{-2}$. The relative error, say $\delta_{a}$ for $a$, is defined as $\delta_{a}=|a(t)-a| / a$ where $a$ is the value of the parameter at the last time step of the numerical evolution.

\section{Numerical evidence for universal dynamics}

The space of solutions to the cubic wave equation can be divided into three parts: decay, blowup and criticality. These parts correspond, respectively, to $b>0, b<0$ and $b=0$ for the attractor solution (7). We will follow this natural classification in our presentation of the numerical evidence for the universality of dynamics.

In many cases, the numerical evidence will be presented in terms of the conformally rescaled solution in the coordinates presented in the previous section. In these variables the two-parameter family of solutions (7) takes the form (using the abbreviation $\tilde{\tau}=\tau+a$ )

$$
\Phi_{(a, b)}(\tau, \rho)=\frac{2 \sqrt{2}}{(\tilde{\tau}+1)(b(\tilde{\tau}+1)+1)-\rho^{2}(\tilde{\tau}-1)(b(\tilde{\tau}-1)+1)} .
$$

\subsection{Decay}

5.1.1. Convergence to the attractor. According to conjecture 1, the difference between the attractor solution (with optimal parameters) and a generic solution should decay as $t^{-4}$ for small data (16). This behaviour is confirmed numerically in figure 4.

For this plot, we first fit the numerical solution to the attractor (25) to determine the optimal parameters $(a, b)$. We applied two methods for the fit. Fit in time and fit in space. For the fit in time we fix a grid point $\rho=\rho_{\mathrm{f}}$, fit the solution $\Phi\left(\tau, \rho_{\mathrm{f}}\right)$ in $\tau$ and repeat the process for each grid point $\rho_{\mathrm{f}}$. This gives us a set of values $(a, b)_{\rho_{\mathrm{f}}}$ depending on $\rho_{\mathrm{f}}$. The average of these values over all grid points gives the optimal parameters. For this method, the starting time for the fit needs to be taken well behind the approximate wavefront because the convergence of the numerical solution to the attractor family, and therefore the variation of $(a, b)_{\rho_{\mathrm{f}}}$ over the grid, will depend strongly on the foliation in early times. Note that our grid has infinite physical extent due to compactification. The variation is expected to decrease in time which we have checked numerically. Choosing a large value of $\tau$ as the starting time of the time fit ensures higher accuracy in the determination of the optimal parameters.

The second method that we applied is the fit in space in which we fix a time $\tau=\tau_{\mathrm{f}}$, fit the solution $\Phi\left(\tau_{\mathrm{f}}, \rho\right)$ in $\rho$ and repeat the process on each time surface $\tau_{\mathrm{f}}$. This gives us a set of values $(a, b)_{\tau_{\mathrm{f}}}$ depending on $\tau_{\mathrm{f}}$. The resulting time variation of the parameters is commonly referred to as modulation. If our conjecture is correct, the modulation should be small. 

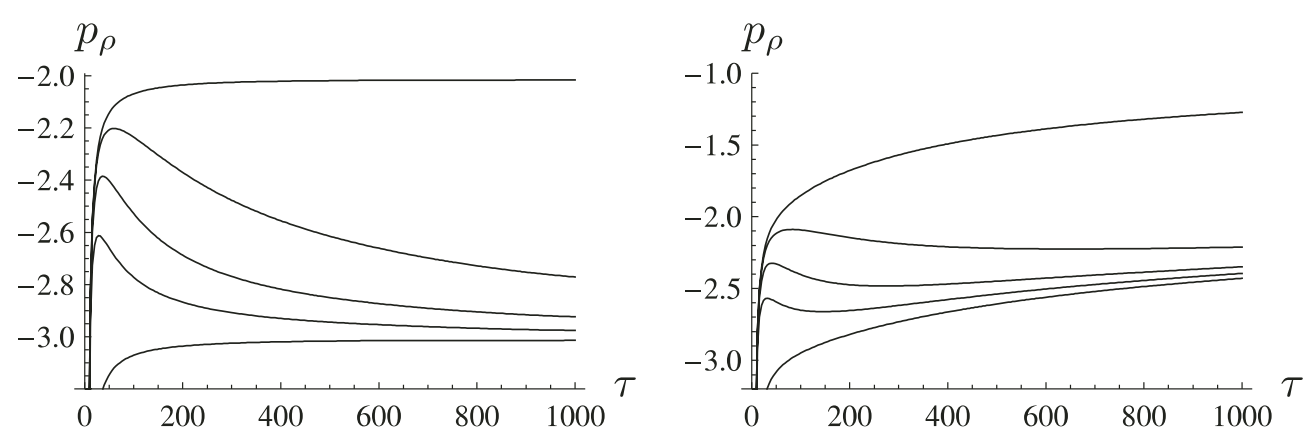

Figure 5. On the left panel we plot decay rates for the flip solution with $A_{\mathrm{f}}=2.4913$ along the surfaces $\mathscr{I}^{+}, r=\{100,33,14,0\}$ from top to bottom. On the right panel we plot the decay rates for a solution with initial amplitude $A_{\mathrm{f}}-0.01$ along the same surfaces. Here, the generic decay rates are obtained after a much longer time than that in figure 3.

At the end, both methods should deliver the same values up to a small error. This is used as a cross-check for the quality of fitting. The modulation of the parameters $a$ and $b$ in time is shown on the right panel in figure 4 . We observe that the accuracy of the fitting in $b$ is better than in $a$. This is due to the fact that the perturbation generated by changing $a$ decays in time while the perturbation generated by changing $b$ does not (14), hence an error in determining $b$ is easier to spot.

Once the optimal parameters have been determined, we compute the difference between the attractor solution and the numerical solution at each time step. The difference $\Phi-\Phi_{(a, b)}$ at late times has a strict sign for all values of $\rho$ on our numerical grid. Therefore, the $L_{2}$-norm of this difference over the grid as a function of time gives a strong uniform measure of how close the solutions are to each other. Figure 4 indicates that this difference falls off as $t^{-4}$, as claimed in conjecture 1 .

5.1.2. Exceptional solutions. As mentioned above in remark 3, not all globally regular solutions converge to the attractor $\phi_{(a, b)}$. The existence of exceptional solutions with different asymptotic behaviour can be seen as follows. Consider a one-parameter family of initial data, such as (24). It turns out that along such a family typically there occurs a flip of sign of the attractor, that is, there is a (subcritical) amplitude $A_{\mathrm{f}}$, such that for $A<A_{\mathrm{f}}$ the solutions converge to, say, $\phi_{(a, b)}$ and for $A>A_{\mathrm{f}}$ they converge to $-\phi_{(a, b)}$. Performing a bisection we may easily fine-tune the amplitude to $A_{\mathrm{f}}$ and generate a special solution (below referred to as the flip solution) that is not an element of the attractor family. One can expect that the flip solution will have a faster decay rate than the generic global solutions. This expectation is confirmed in figure 5 which shows that the flip solution decays as $t^{-3}$ at timelike infinity and as $t^{-2}$ along null infinity. By modifying the initial amplitude slightly away from the flip solution, we can see that the decay rates of the generic solutions are attained at late times after a transient phase. Note that by construction the flip solutions correspond to codimension-one initial data. It is likely that there exist initial data of higher codimensions which lead to globally regular solutions with even faster decay rates; however, the numerical construction of such solutions would be very difficult.

\subsection{Blowup}

5.2.1. Convergence to the attractor. Merle and Zaag proved in [3] that the ODE solution $\sqrt{2} /(T-t)$ determines the universal rate of blowup for equation (1); however, the problem 


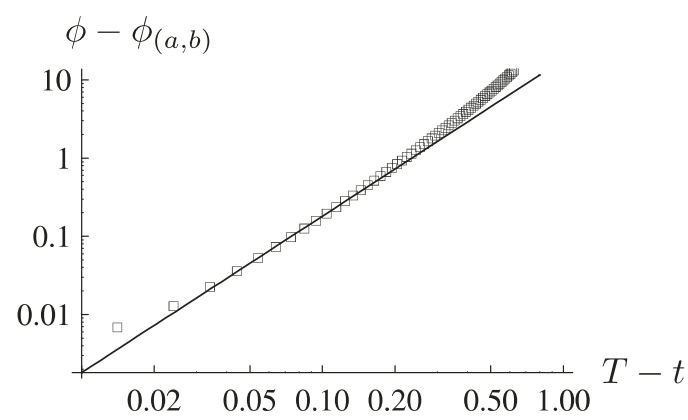

Figure 6. Blowup at the origin in standard coordinates on a $\log -\log$ scale. The difference between the attractor solution and the numerical solution is depicted by small squares. The linear fit (solid line) gives the slope 2.02, which confirms conjecture 2 . The deviation from the straight line seen at times very close to the blowup time is due to the fact that the domain of analysis extends beyond the past light cone of the blowup point.

of profile of blowup remains open. Numerical simulations in spherical symmetry [4] showed that for a solution which blows up at the origin, its deviation from $\sqrt{2} /(T-t)$ near the tip of the past light cone of the blowup point is very well approximated by the second eigenmode in the expansion (12) (see figure 4 in [4])

$$
\phi(t, r)-\frac{\sqrt{2}}{T-t} \approx c_{1}\left(1-\frac{r^{2}}{(T-t)^{2}}\right) ;
$$

however, an error on the right-hand side was not quantified in [4]. According to conjecture 2 the approximation (26) may be improved to

$$
\phi(t, r)-\phi_{(a, b)}(t, r) \approx \mathcal{O}\left((T-t)^{2}\right),
$$

provided that the parameters $(a, b)$ are optimal.

The numerical verification of formula (27) is shown in figure 6. The data for this plot were produced using a code based on standard Minkowski coordinates.

We point out that there is no genericity condition in conjecture 2. This might appear surprising in view of the existence of a countable family of regular self-similar solutions of equation (2) [14]. It seems that these self-similar solutions do not play any role in the Cauchy evolution, which is probably due to the fact that they contain singularities outside the past light cone of the blowup point (see section 6 in [14]).

5.2.2. Blowup surface. For $b<0$ the attractor solutions blow up along a hyperboloid

$$
t=-a-\frac{1}{2 b}+\sqrt{\frac{1}{4 b^{2}}+r^{2}}
$$

This surface has the form (20) with the mean extrinsic curvature $K=-6 b$. Hence, for our choice of the hyperboloidal foliation with $K=3$ the blowup surface of the attractor with $b=-1 / 2$ coincides with one leaf of the foliation. Therefore, if conjecture 2 is correct, in the case of the blowup solution converging to the attractor with the optimal parameter $b=-1 / 2$ we should observe an approximately simultaneous blowup along the whole grid. This expectation is verified in figure 7.

Note that, for a given constant mean curvature foliation, the simultaneous blowup is not generic. To produce data for figure 7 we used the dependence of $b$ on the amplitude $A$ of the Gaussian. For relatively small (but supercritical) values of $A$ we have $-1 / 2<b<0$ and 


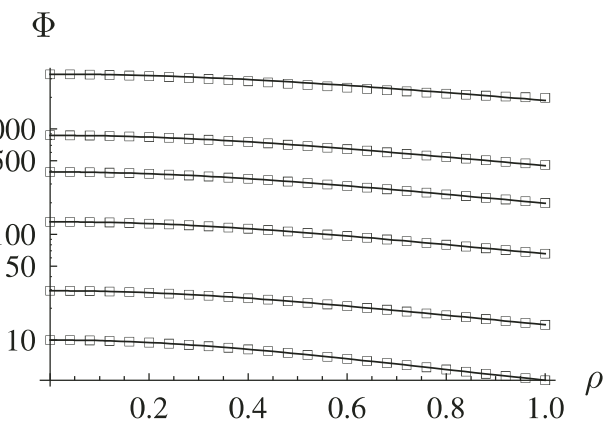

Figure 7. Simultaneous blowup along the numerical grid for fine-tuned initial data. The solution is plotted on a $\log$ scale at various time steps close to the blowup time $T$. The solid lines depict an attractor solution with $b=-1 / 2$ at the corresponding times. The times are from bottom to top $\triangle T=T-\tau=\{0.34,0.1,0.02,0.007,0.003,0.0008\}$.

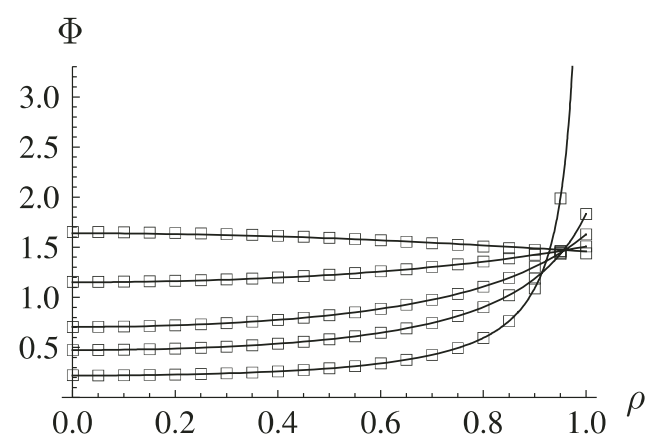

Figure 8. Blowup at null infinity. The numerical solution (small squares) at the times (counting, near the origin, from top to bottom) $\tau=\{2.3,3.2,5,7,25\}$ is compared with the attractor solution (solid line) with $b=-0.02$. We see that the solution grows at null infinity while it decays near the origin.

the blowup first occurs at null infinity, while for larger amplitudes we have $b<-1 / 2$ and the blowup first occurs at the origin. Performing bisection between these two states we fine-tuned to the blowup solution with $b=-1 / 2$.

We would like to emphasize that a rather counter-intuitive phenomenon of blowup at null infinity is a mere coordinate effect. This only occurs if the mean extrinsic curvature of the blowup surface, given by $-6 b$, is smaller then the mean extrinsic curvature of the hyperboloidal foliation, $K$, used in the numerical simulation. Nevertheless, as discussed above, we can use this effect to our advantage to probe the shape of the blowup surface. Note also that one can predict the blowup at null infinity by fitting the attractor to the numerical solution at the origin. If this fit gives a value of $b \in(-1 / 2,0)$, then we know that the solution will blow up at null infinity even though it is decaying at the origin (see figure 8 ).

\subsection{Critical behaviour}

Now we consider the behaviour of solutions for initial data lying at the boundary between dispersion and blowup. Let us recall that this problem was addressed before in [4] for the focusing wave equation $\partial_{t t} \phi-\Delta \phi-\phi^{p}=0$ with three values of the exponent $p$ (corresponding to three different criticality classes with respect to scaling of energy): $p=3$ (subcritical), 

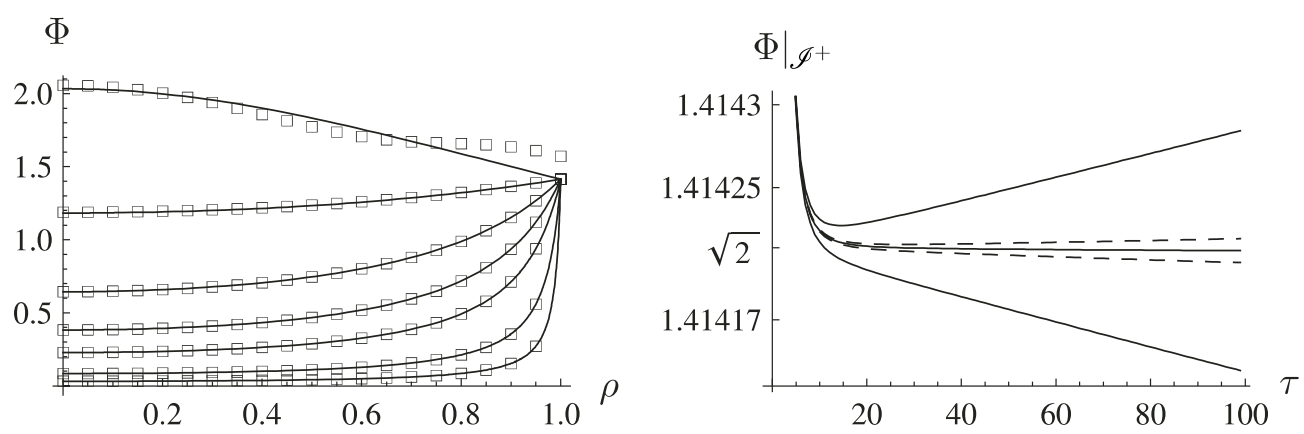

Figure 9. Left panel: the critical solution on the grid at times $\tau=\{2,3,5,8,13,34,89\}$ (counting from top to bottom). We see that at $\tau=2$ the solution is not yet described well by the attractor solution, but already after $\tau=3$ the agreement is good. Right panel: numerical solutions close to the critical solution evaluated at null infinity. Denoting the initial amplitude for the solution that corresponds to the almost constant solid line at $\sqrt{2}$ by $A_{\mathrm{c}}$, the deviating dashed curves have $A=A_{\mathrm{c}} \pm 10^{-9}$, the deviating solid ones have $A=A_{\mathrm{c}} \pm 10^{-8}$.

$p=5$ (critical) and $p=7$ (supercritical). It was shown there that the nature of the critical solution, whose codimension-one stable manifold separates blowup from dispersion, depends on $p$ : for $p=7$ the critical solution is self-similar, while for $p=5$ it is static. For $p=3$ the critical solution was not determined because of numerical difficulties (although in hindsight it could have been inferred from figure 11 in [4]). Now, in view of conjectures 1 and 2 which assert that the solution $\phi_{(a, b)}$ is an attractor for generic dispersive solutions if $b>0$ and for blowup solutions if $b<0$, it is easy to guess that the critical solution corresponds to $b=0$, hence it has the form $\phi_{\mathrm{c}}=\sqrt{2} /(t+a)$.

The critical solution is difficult to study with standard numerical methods by bisection because it is a globally decaying solution, but it can be studied very accurately with the conformal method. The reason is that the conformal scaling factors out the leading asymptotic behaviour implying that the $1 / t$ decay of the critical solution is factored out at the conformal boundary. Specifically, the rescaled critical solution $\Phi_{\mathrm{c}}=\phi_{\mathrm{c}} / \Omega$ in the new coordinates as given in (25) evaluated at null infinity becomes $\left.\Phi_{\mathrm{c}}\right|_{\mathscr{I}^{+}}=\Phi_{(a, 0)}(\tau, 1)=\sqrt{2}$, hence the deviation of $\left.\Phi\right|_{\mathscr{I}^{+}}$from $\sqrt{2}$ can be used as a bisection criterion. In the left panel of figure 9 we plot the critical solution on the grid at various time steps and compare it with the attractor solution with $b=0$ and fitted $a$. We see that the solution is decaying while its value at $\mathscr{I}^{+}=\{\rho=1\}$ is constant. The instability of the critical solution can be seen by evolving initial data that differ slightly from the critical amplitude $A_{\mathrm{c}}$-for such data the deviation from $\sqrt{2}$ at $\mathscr{I}^{+}$grows linearly with time. This is depicted in the right panel of figure 9 for four different values of marginally critical amplitude.

\section{Final remarks}

We wish to emphasize that the use of the hyperboloidal foliation (20) in combination with the conformal method was instrumental in unravelling the dynamics of global solutions of equation (2). First and foremost, this method eliminates the need for introducing an artificial boundary, which is a notorious problem in computing wave propagation on unbounded domains. Second, the intersection of $\tau=$ const hyperboloids with $\mathscr{I}^{+}$increases monotonically with $\tau$ leading to the dispersive dissipation of energy along the leaves of the foliation which is a mechanism responsible for convergence to the attractor. Third, the conformal rescaling allows a very 
accurate computation of the critical solution by factoring out its leading asymptotic behaviour. Finally, with this approach we can probe efficiently the shape of the blowup surface and observe the simultaneous blowup on the whole grid.

Our main observation that a simple family of exact solutions can act as a universal attractor for solutions of the nonlinear wave equation was unexpected to us. It is clear that this surprising phenomenon is intimately related to the conformal invariance of the cubic wave equation, and therefore it is more a curiosity than a stable property; in particular, it is absent for semilinear focusing wave equations

$$
\square \phi+|\phi|^{p-1} \phi=0
$$

with $p \neq 3$. However, we conjecture that the threshold behaviour mediated by the slowly decaying global solution is structurally stable in the sense that the ODE solution of equation (29)

$$
\phi=\frac{c}{(t+a)^{\frac{2}{p-1}}}, \quad \text { where } \quad c=\left[\frac{2(p+1)}{(p-1)^{2}}\right]^{\frac{1}{p-1}},
$$

is a critical solution for all exponents satisfying $1+\sqrt{2}<p \leqslant 3$. Note that the decay rate exponent of the critical solution, $2 /(1-p)$, and the decay rate exponent of generic solutions, $1-p$, merge for $p \rightarrow 1+\sqrt{2}$, which is consistent with the fact that for $p \leqslant 1+\sqrt{2}$ all solutions of equation (29) with compactly supported initial data blow up in finite time [15].

\section{Acknowledgments}

The authors thank Helmut Friedrich, Sascha Husa, Vince Moncrief and Nikodem Szpak for discussions. PB thanks the Albert Einstein Institute in Golm for hospitality and support during the initial phase of this project. PB acknowledges support by the MNII grants: NN202 079235 and 189/6.PRUE/2007/7. AZ acknowledges support by the NSF grant PHY0801213 to the University of Maryland.

\section{References}

[1] Christodoulou D 1986 Commun. Pure Appl. Math. 39 267-82

[2] Szpak N, Bizoń P, Chmaj T and Rostworowski A 2009 J. Hyperbolic Diff. Eqns 6 107-25 (arXiv:0712.0493)

[3] Merle F and Zaag H 2005 Math. Ann. 331 395-416

[4] Bizoń P, Chmaj T and aw Tabor Z 2004 Nonlinearity 17 2187-201 (arXiv:math-ph/0311019)

[5] Anco S C and Liu S 2004 J. Math. Anal. Appl. 297317

[6] Christodoulou D 2008 Mathematical Problems in General Relativity I (Zurich Lectures in Advanced Mathematics) (Switzerland: European Mathematical Society)

[7] Galaktionov V and Pohozaev S 2003 Q. Appl. Math. 61 583-600

[8] Penrose R 1965 Proc. R. Soc. Lond. A 284 159-203

[9] Hawking S W and Ellis G F R 1973 The Large Scale Structure of Spacetime (Cambridge: Cambridge University Press) ISBN 0-521-09906-4

[10] Zenginoğlu A 2008 Class. Quantum Gravity 25145002 (arXiv:0712.4333)

[11] Moncrief V 2000 Conformally regular ADM evolution equations, talk at Santa Barbara anf http://online.itp.ucsb.edu/online/numrel00/moncrief

[12] Husa S 2003 Current Trends in Relativistic Astrophysics (Lecture Notes in Physics vol 617) ed L Fernández and L M González (Berlin: Springer) (arXiv:gr-qc/0204057)

[13] Fodor G and Racz I 2004 Phys. Rev. Lett. 92151801 (arXiv:hep-th/0311061)

[14] Bizoń P, Breitenlohner P, Maison D and Wasserman A arXiv:0712.0493

[15] John F 1979 Manuscript. Math. 28 235-68 